Noman 2016, 34(2), 49-56

Revista de Psicologia, Ciències de l'Educació i de l'Esport

ISSN: 1138-3194

Copyright (c) 2016

www.revistaaloma.net

\title{
Solucions tecnològiques per a l'estudi de la variabilitat de la freqüència cardíaca, l'estrès i la recuperació
}

\author{
José Morales Aznar, Alexandre Yáñez de la Cal \& Mònica Solana-Tramunt \\ Universitat Ramon Llull
}

Rebut:4-5-2016

Acceptat: 15-9-2016

Solucions tecnològiques per a l'estudi de la variabilitat de la freqüència cardíaca, l'estrès i la recuperació

Resum. La variabilitat de la freqüència cardíaca (VFC) fa referència al temps de l'interval entre batec i batec del cor. A causa del fet que quest interval no és fix sinó que oscil-la constantment, la dinàmica d'aquestes oscil-lacions, juntament amb una anàlisi matemàtica adequada, pot aportar una informació molt valuosa sobre l'equilibri del Sistema Nerviós Autònom (SNA) com a responsable del funcionament del cor. L'anàlisi de la VFC aporta informació sobre qualsevol situació que trenqui l'equilibri homeostàtic en el nostre organisme; és per això que la seva variació va associada amb la presència de certes patologies. En l'àmbit de l'entrenament dels esports, atès que l'exercici físic és un agent promotor de l'estrès fisiològic, els canvis de la VFC s'han associat a l'assimilació de les càrregues d'entrenament o a certes condicions vinculades a la síndrome de sobreentrenament. Els avenços tecnològics han aportat solucions per a analitzar la VFC; en aquest sentit es presenten diferents opcions de software científic per a analitzar en profunditat la dinàmica de la VFC, com també diverses opcions d'aplicacions per a dispositius mòbils que cada cop més ocupen aquest espai en el món de l'entrenament esportiu.

Paraules clau: Sistema nerviós autònom; sobreentrenament; Interval inter-batec; equilibri simpàtic-parasimpàtic

Technological solutions for the study of variability in heart rate, stress and recovery

Summary. The term heart rate variability (HRV) refers to the time interval between heartbeats. Because this time interval is not fixed, but rather fluctuates constantly, bringing to bear the right mathematic analysis on these fluctuations can yield highly valuable information on how the autonomic nervous system (ANS) acts in its role of controlling heart functioning. HRV analysis provides information on any situation that may interfere with homeostatic balance in our bodies, with variations associated with the presence of certain pathologies. In the field of sport training, given that physical exercise can cause physiological stress, changes in HRV have been associated with the assimilation of training loads and with certain conditions linked to overtraining syndrome. Technological advances have provided solutions for the analysis of HRV. Thus, this article presents some scientific software options that can be used to conduct in-depth analyses of the dynamics of HRV, as well as several mobile apps that have been taking on greater and greater roles in this area of the world of sport training.

Keywords: autonomic nervous system; overtraining; heartbeat interval; sympathetic-parasympathetic balance

Correspondència:

José Morales Aznar FPCEE Blanquerna

Universitat Ramon Llull

Carrer Císter, 34

08022 Barcelona

josema@blanquerna.edu 


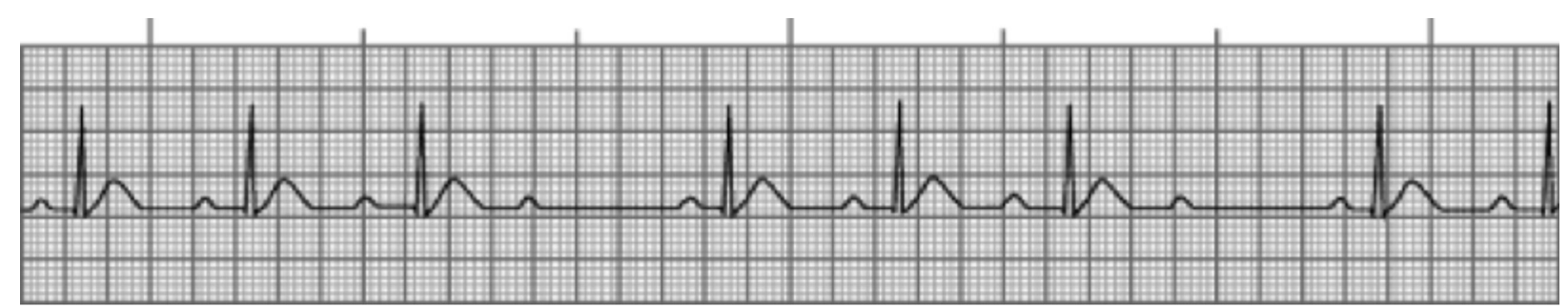

Figura 1. Activitat elèctrica del cor detectada per un ECG. Les ones més altes són les ones R i l'espai de temps entre les unes i les altres és el temps entre batec i batec.

\section{Introducció}

L'objectiu principal d'aquest article és introduir els conceptes bàsics de la Variabilitat de la Freqüència Cardíaca (VFC) i explicar les aplicacions tecnològiques utilitzades en la seva anàlisi. La VFC fa referència al fenomen fisiològic en el qual el temps entre batec $\mathrm{i}$ batec va oscil-lant contínuament.

El concepte de Freqüència Cardíaca (FC) és tan comú que sovint es confon amb el que ens ocupa en aquest article: la VFC. La FC es mesura comptant el nombre de batecs del cor en un temps determinat; per exemple, 60 batecs/min. Amb aquesta dada tendim a pensar que el temps entre batec i batec és d'un segon; la realitat ens demostra que no és així, ja que el temps entre batec i batec pot oscil-lar molt: podem trobar intervals de 0,5 seg a 1,5 seg i, fins i tot, diferències més grans. Aquesta irregularitat es coneix com a VFC.

Per tant, no podem pensar que el cor batega a un ritme fix; l'interval entre batec i batec és molt variable. Concretament estem parlant de la mesura del temps entre intervals RR. Normalment en l'àmbit clínic es fa amb un electrocardiograma (ECG) i es veu, representat pel pic, la part més alta de les ones de la gràfica generada per l'ECG (Figura 1).

La VFC proporciona informació en relació al funcionament del sistema nerviós autònom (SNA). Mentre que el sistema nerviós central (SNC) és responsable de tots els moviments voluntaris, el SNA contribueix al control de tota l'activitat vegetativa (evidentment involuntària), participant en l'homeòstasi fisiològica a través de la modulació hormonal i el control de la musculatura llisa cardíaca.

El SNA es compon de dues branques: el sistema nerviós simpàtic (SNS), al qual s'associen diferents atributs com «activació», «lluita» $\mathrm{O}$ «fugida», i el sistema nerviós parasimpàtic (SNPS), amb atributs com «tornada a la calma», «descans» 0 «digestió». L'activitat simpàtica proporciona al cos tots els recursos per a respondre a una situació d'estrès (augment de la FC, augment de la força de contracció muscular, augment de la pressió arterial, disminució del flux sanguini en els òrgans gastrointestinals). En contrast, l'activació parasimpàtica fa tot el contrari. En absència d'estrès, el cos reduirà el seu ritme cardíac i la pressió arterial, augmentarà els processos digestius i se centrarà en l'acumulació i la reparació de teixits (com el múscul) i emmagatzematge de sucre (glicogen). En essència, el cos es recupera de qualsevol dany causat durant la resposta a l'estrès del SNS.
La FC està controlada, en gran part, per la influència simpàtica i per la parasimpàtica que s'originen en el centre cardiovascular del cervell. El centre cardiovascular està situat a la part inferior del tronc de l'encèfal, en el bulb raquidi. Des d'aquí, les neurones simpàtiques s'estenen des del cervell, a través de la medul-la espinal, i van directament al cor (Figura 2).

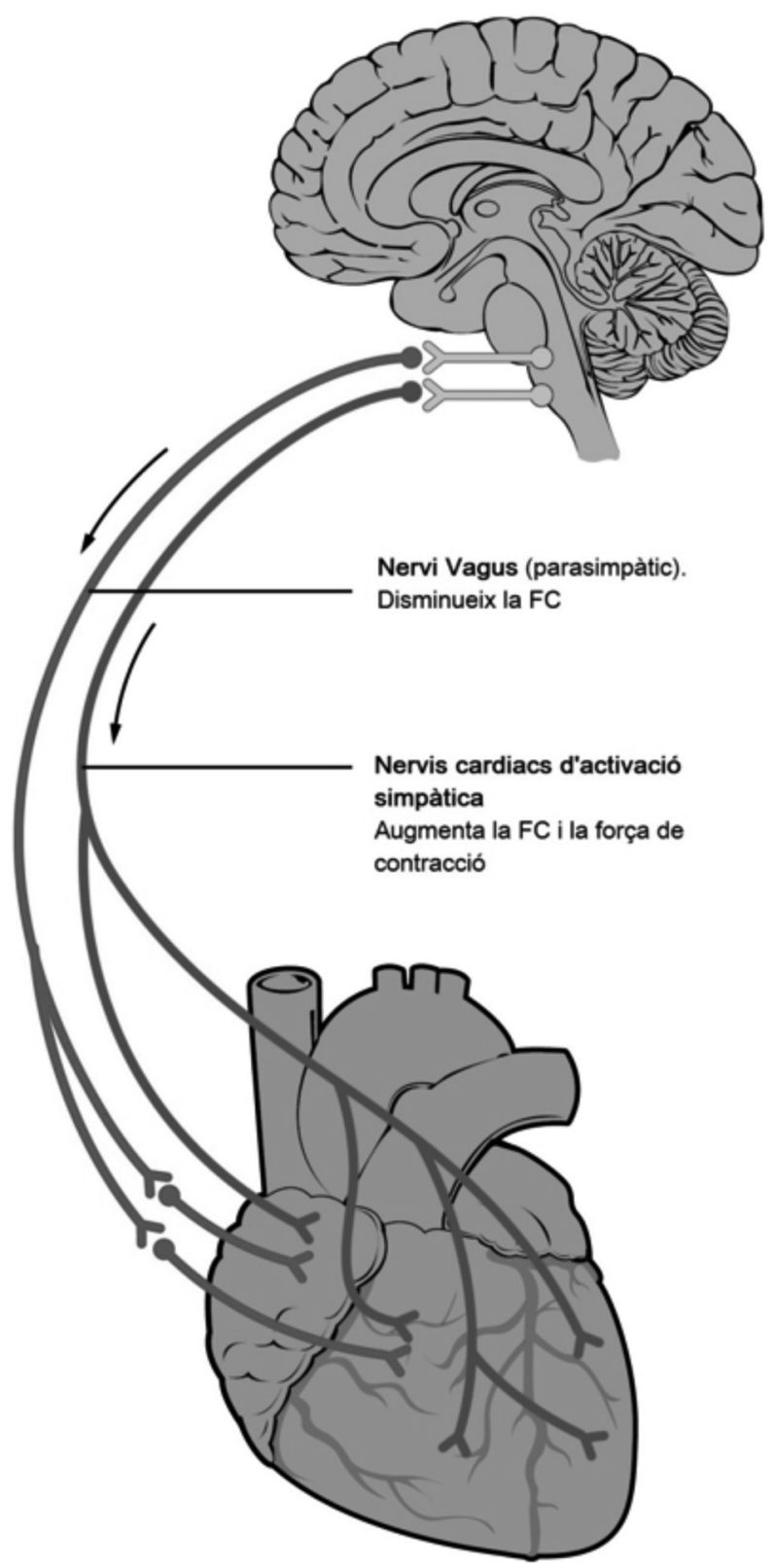

Figura 2. Esquema que representa la innervació del cor. Adaptat d'OpenStax, Anatomy and Physiology. OpenStax. 25 d'abril de 2016. http://cnx.org/content/col11496/latest/. 
La influència parasimpàtica del cor es produeix a través del nervi vague (10è nervi cranial), que s'origina al bulb raquidi i té axons que acaben directament al cor. L'estimulació vagal provoca un efecte inhibidor en el nòdul sinoatrial (SA) a través de l'alliberament d'acetilcolina. Atès que l'activitat vagal inhibeix l'activitat del nòdul SA, la retirada vagal es traduirà en una menor inhibició nòdul SA i permetrà que el cor bategui més ràpid. A l'inici de l'exercici, l'increment inicial de la FC és el resultat de la retirada vagal amb un augment progressiu de l'activitat simpàtica a mesura que persisteix l'exercici (Yamamoto, Hughson, \& Peterson, 1991).

\section{Aplicacions de la VFC}

L'activitat del SNA afecta directament la VFC; per tant, la dinàmica d'aquesta última pot ser un bon marcador de l'equilibri simpàtic-parasimpàtic i de l'estat del SNA. L'observació de la VFC és relativament senzilla; no tant la seva interpretació. En termes generals, està acceptat que un augment de la VFC sigui un indicador de bona salut, tal com indica el Cardiovascular Health Study (Stein et al., 2008), en el qual s'interpreta que una major irregularitat entre batec i batec indicaria que el nostre sistema cardiovascular s'adapta millor a qualsevol esforç o situació d'estrès. Segons Rodas, Pedret, Ramos, \& Capdevila, (2008b), l'observació de la VFC té una enorme utilitat en diferents àmbits clínics que faciliten el seu control i prevenció; per exemple, una disminució dels seus paràmetres està associada a síndromes de fatiga crònica, trastorns d'hipertensió arterial, major risc en patologies cardíaques i major risc de mort sobtada en malats de Diabetis Mellitus.

L'anàlisi de la VFC també pot donar informació sobre molts aspectes psicosomàtics que tenen el seu origen en l'ansietat i l'estrès (D'Ascenzi et al., 2014; J. Morales et al., 2013) i darrerament n'estan apareixent moltes altres en les quals s'analitza el burn-out a partir de les dades proporcionades per la VFC (Hynynen, Konttinen, Kinnunen, Kyröläinen, \& Rusko, 2011; Kotov \& Revina, 2012).

L'àmbit de l'entrenament esportiu també s'està beneficiant del gran volum de recerca al voltant de la VFC. En aquest sentit, un dels primers estudis va ser el desenvolupat per Aubert, Seps i Beckers (2003), en el qual es va comparar els paràmetres de VFC entre subjectes en- trenats i no entrenats i es va demostrar que els primers tenen una major VFC I, per tant, un sistema cardiovascular més ben adaptat a qualsevol demanda. Posteriorment alguns estudis es van focalitzar a mesurar la VFC durant l'exercici a diferents intensitats (Cottin et al., 2004; Pichon, de Bisschop, Roulaud, Denjean i Papelier, 2004), però es va crear certa controvèrsia a causa del fet que molts investigadors defensaven que les condicions d'adquisició de les dades de VFC havien de ser en condicions estacionàries (Kaikkonen, Nummela i Rusko, 2007a), ja que, sinó, elements com la respiració o l'augment de la pressió sanguínia podien emmascarar els resultats. Després d'aquest període van aparèixer moltes investigacions centrades en l'anàlisi de la VFC durant la recuperació immediata postexercici, buscant unes condicions estacionàries, observant la seva evolució durant la recuperació I, d'aquesta manera, poder valorar l'efecte de diferents càrregues en diferents tipus d'atletes (Kaikkonen, Hynynen, Mann, Rusko i Nummela, 2010, 2012; Kaikkonen, Nummela i Rusko, 2007b; Seiler, Haugen i Kuffel, 2007). Per últim, la tendència dels darrers temps és intentar detectar situacions de sobreentrenament en atletes d'alt nivell (Dupuy, Bherer, Audiffren i Bosquet, 2013; Morales et al., 2014; Sartor, Vailati, Valsecchi, Vailati i La Torre, 2013; Thiel et al., 2011) i d'aquesta manera poder preveure possibles conseqüències negatives en la feina de molts entrenadors.

\section{Aplicacions tecnològiques en l'anàlisi de la VFC}

Actualment, qualsevol esportista ocasional disposa d'instruments que l'ajuden a controlar una multitud de paràmetres relacionats amb l'activitat física desenvolupada: des de podòmetres, acceleròmetres, samarretes d'activitat $\mathrm{o}$, fins i tot, els clàssics pulsímetres que cada cop incorporen més funcions. Fa gairebé una dècada que els pulsímetres d'alta gamma de marques com Polar ${ }^{\mathrm{TM}}$ o Suunto ${ }^{\mathrm{TM}}$ incorporen la possibilitat de mesurar el temps entre batec i batec, moltes vegades anomenat interval $\mathrm{RR}$, fent referència al temps entre cada ona R de l'ECG, o simplement, amb l'acrònim IBI de l'anglès (inter-beat interval). Durant aquest procés cal destacar l'aparició de diversos articles confirmant la validesa d'aquestes dades, comparades amb les obtingudes amb un ECG (Gamelin, Berthoin i Bosquet, 2006; Nunan et al., 2009; Weippert et al., 2010) (Figura 3).
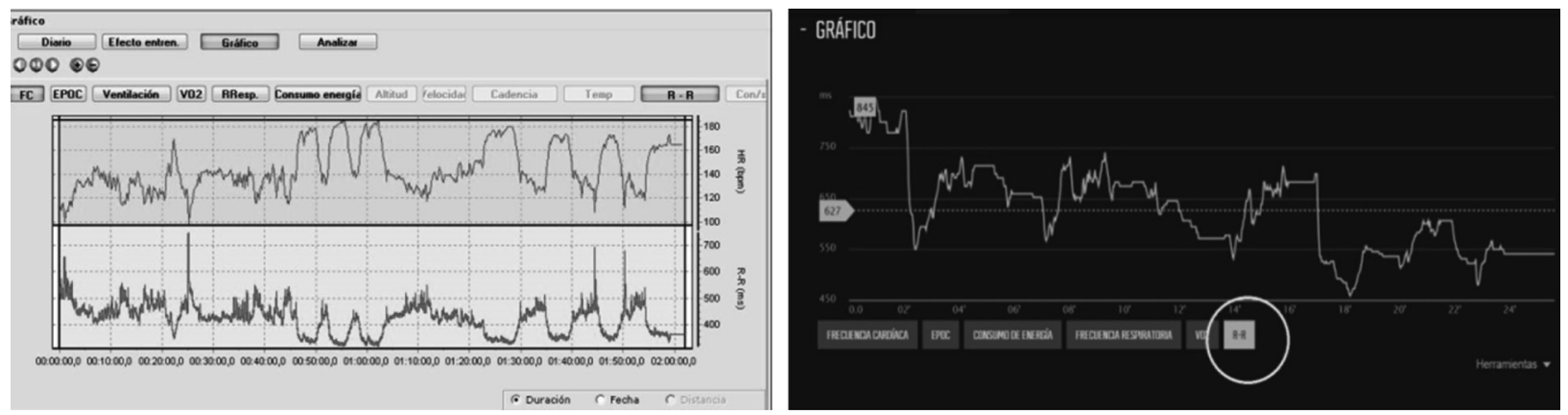

Figura 3. Exemples de tacogrames de la funció cardíaca registrada per pulsímetres. 


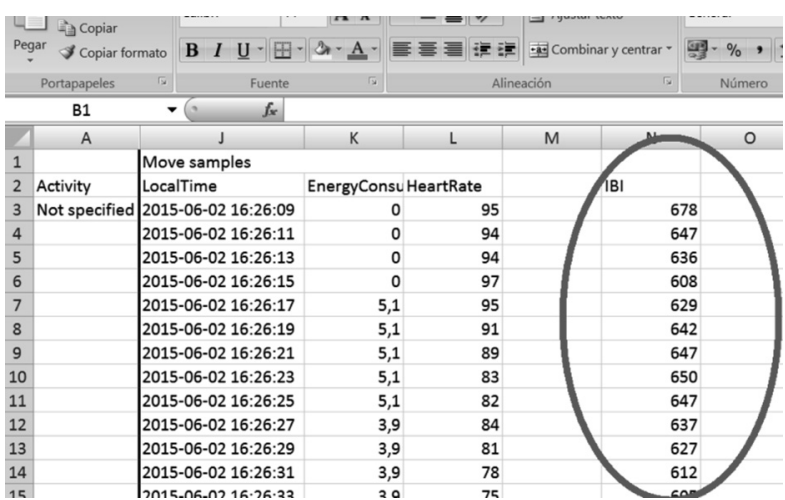

Figura 4. Exemple de full $d^{\prime}$ Excel $^{\mathrm{TM}}$ exportat d'un programa de gestió de pulsímetres.

El temps entre batec i batec (RR) es pot representar mitjançant un tacograma, que ens proporcionarà una visualització gràfica de la VFC. Una característica d'aquests instruments és que disposen de plataformes per a poder consultar la informació i, sobretot, per a facilitar l'exportació en arxius de diferents formats (xlsx, .xlm, .txt, .csv...) (Figura 4).

L'evolució de les aplicacions de la VFC en diferents àmbits, la facilitat en l'obtenció de dades i l'augment exponencial de publicacions científiques en aquest àmbit ha fet proliferar un gran volum de software científic, normalment d'utilització lliure, desenvolupat moltes vegades en grups de recerca universitaris. Els sistemes més comuns per a analitzar les sèries d'inter- vals utilitzen mètodes estadístics i funcions matemàtiques de més o menys complexitat, centrats normalment en el domini temporal, domini freqüencial i l'anàlisi no lineal. L'anàlisi del domini temporal aporta informació sobre diferents paràmetres resultants del mesurament dels intervals RR i la seva anàlisi estadística. Les dades del domini freqüencial s'obtenen a partir de l'estimació de la densitat de la potència espectral del senyal RR. Les mesures no lineals de la HRV aporten una informació qualitativa molt important, ja que mesuren l'estructura i la complexitat dels intervals RR (Rodas, Pedret, Ramos i Capdevila, 2008a).

En la taula 1 s'exposen els paquets de software més utilitzats per a l'anàlisi de la VFC en l'àmbit científic (Taula 1). El procediment per a realitzar aquesta anàlisi és tan senzill com exportar l'arxiu guardat en un pulsímetre i obrir-lo amb qualsevol d'aquests programes.

En el domini temporal de la VFC s'obtenen els valors següents: la mitjana de l'interval R-R (MEANRR), la desviació típica del R-R (STDRR), la freqüència cardíaca (HR), la desviació típica de la freqüència cardíaca (STDHR), l'arrel quadrada de la mitjana de la suma de les diferències al quadrat de tots els R-R (RMSSD), el nombre de R-R consecutius que difereixen més de $5 \mathrm{~m}$ entre ells (NN50), el percentatge de R-R consecutius que difereixen més de $5 \mathrm{~m}$ entre ells (pNN50). Per a l'anàlisi del domini freqüencial de la HRV s'utilitzen les dades obtingudes per la transformada ràpida de Fourier (FFT), que permet obtenir informació sobre el balanç simpà-

Taula 1. Llista de programes de software per a analitzar la VFC

KUBIOS $^{\mathrm{TM}}$. http://kubios.uef.fi/

És possiblement el sistema més utilitzat i amb el qual s'han publicat més articles científics. Desenvolupat per investigadors de la Universitat de Kuopio (Finlàndia), permet l'anàlisi en el domini temporal, freqüencial i no lineal, com també la selecció de sèries temporals i l'eliminació de batecs ectòpics.

Es pot descarregar de manera gratuïta.
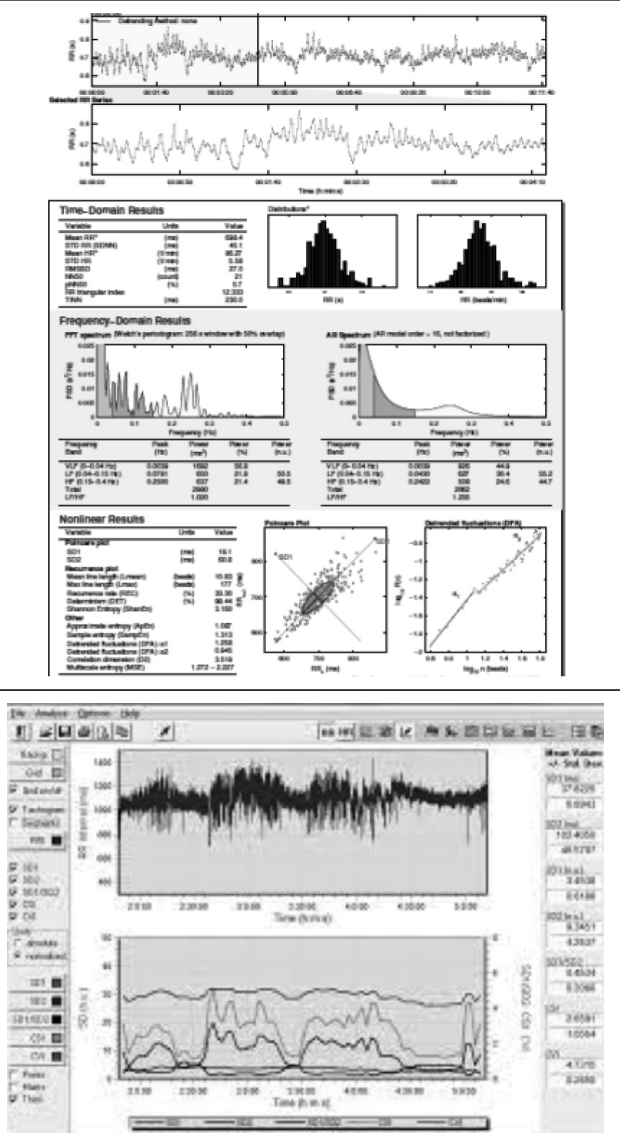

Després de Kubios és el següent programa en difusió, desenvolupa per investigadors eslovens; té les mateixes funcions que Kubios, però, en diferents àmbits, el consideren més fiable, tot i que s'han trobat diferències significatives en els seus resultats.

És un software de pagament i l'ofereixen d'una manera gratuïta durant un temps. 
Taula 1. Llista de programes de software per a analitzar la VFC (continuación)

CODESNA $^{\mathrm{TM}}$. http://www.codesna.com/fr/

És el software d'aparició més recent, desenvolupat per investigadors francesos. L'avaluació de la VFC la fan a partir de l'estimulació de la respiració a través d'estímuls visuals. Els algoritmes d'aquest programa només necessiten $2 \mathrm{~min}$. de temps registrat, mentre que la resta necessiten normalment 5 minuts si volen complir amb els requisits marcats (Task Force, 1996).
ARTiiFACT $^{\mathrm{TM}}$. http://www.artiifact.de/

És un software gratuït desenvolupat per investigadors alemanys i noruecs, de fâcil utilització i que analitza les dades en el domini temporal i freqüencial.
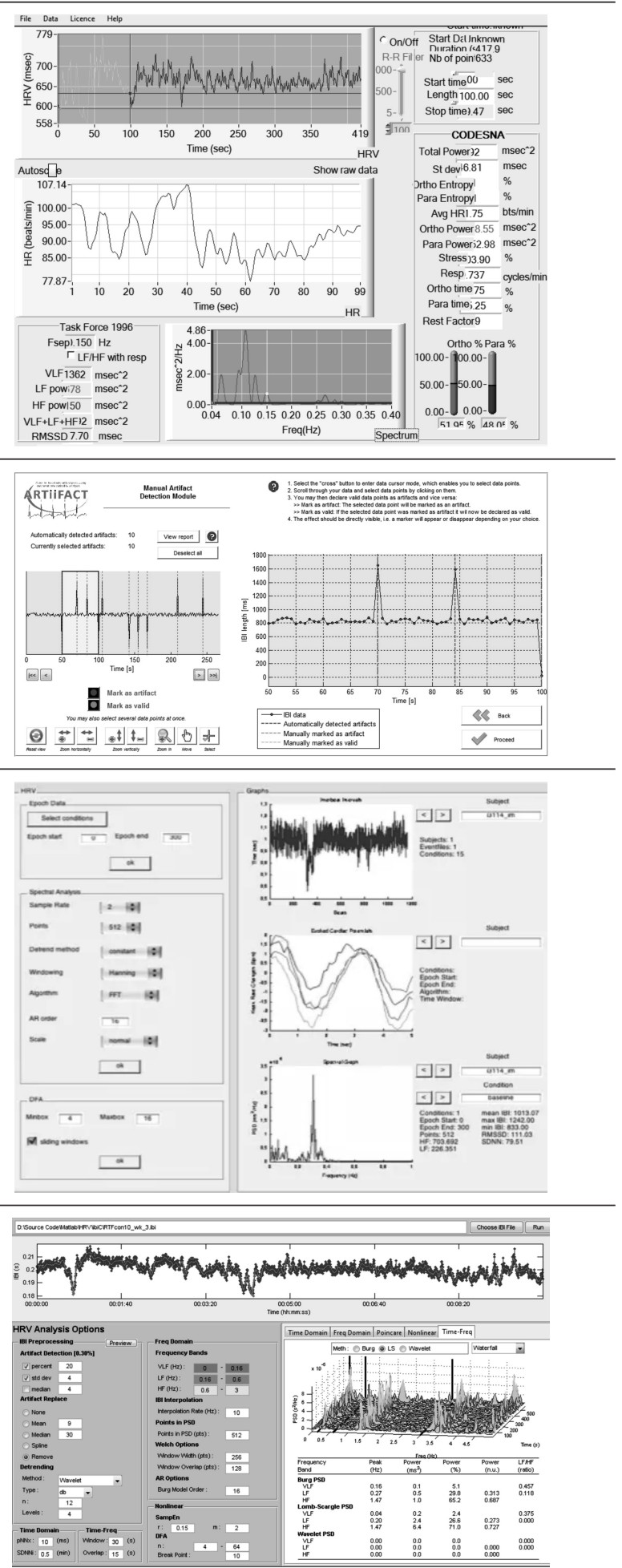

tic-parasimpàtic del sistema nerviós autònom (SNA). La resposta espectral aportada pel sistema es descompon en tres bandes: Molt Baixa Freqüència (VLF, 0,003,04 Hz); Baixa Freqüència (LF, 0,04-0,15 Hz) i Alta Freqüència (HF, 0,15-0,4 Hz). La proporció LF/HF és una mesura per a estimar l'equilibri simpàtic-vagal. Les tècniques d'anàlisi no lineal utilitzades més sovint per aquests programes són aquestes: el Diagrama de Poincaré i les Detrended Fluctuation i diversos indicadors de l'entropia, que es podria dir que són uns indicadors que mesuren el grau d'organització del sistema analitzat (Task Force, 1996).

Si observem la tendència d'aquests paràmetres, podrem estimar l'equilibri del SNA, és a dir, si hi ha un predomini del sistema simpàtic o del parasimpàtic. En cas d'un augment del sistema vagal o parasimpàtic, es produeix una disminució del sistema simpàtic $i$, en conseqüència, un augment de la VFC. Com a guia bàsica per a interpretar aquests paràmetres, podem apuntar que, quan augmenten paràmetres del domini 
temporal com pNN50, STDRR, RMSSD, vol dir que hi ha un augment de l'activitat parasimpàtica i, per tant, un augment de la VFC. En el cas dels paràmetres del domini freqüencial, l'augment de la VFC es produeix amb un augment de HF i LF/HF i descens en LF.

Per altra banda, a part del software científic per a analitzar la VFC, s'han desenvolupat una sèrie d'aplicacions per a dispositius mòbils que utilitzen l'anàlisi de la VFC per a diverses funcions. Aquestes aplicacions basen la seva anàlisi en la utilització d'un paràmetre del domini temporal com és el RMSSD; aquest paràmetre es fa servir normalment com a indicador de l'activitat del nervi vague (activitat parasimpàtica) i el seu avantatge és que és estable en mesures ultracurtes (Plews, Laursen, Stanley, Kilding i Buchheit, 2013), és a dir, en mesures de menys d'un minut, aspecte que millora la funcionalitat d'aquestes aplicacions respecte als programes de software científics anteriors que moltes vegades necessiten registres de 5 minuts, importar l'arxiu i analitzar-lo en un software a part.

Les aplicacions per a dispositius mòbils per a analitzar la VFC són les següents:

\section{- FITLAB}

https://www.healthsportlab.com/fitlab_supervisor. html

És una aplicació desenvolupada per investigadors de la Universitat Autònoma de Barcelona (UAB) i controla variables que influeixen en el rendiment esportiu com l'estrès i la recuperació, l'estat d'ànim, etc. És l'única disponible en els idiomes català, castellà i anglès.

\section{- ITHLETE}

http://www.myithlete.com/

Possiblement es tracta de l'aplicació més utilitzada en l'àmbit de l'esport i la que s'està utilitzant més per a publicacions científiques; de fet, hi ha una publicació que valida la seva utilització (Flatt \& Esco, 2013). Aquesta aplicació utilitza el logaritme natural transformat de RMSSD multiplicat per 20 (lnRMSSDx20) per tal de construir una escala de fàcil interpretació. A part de la banda pectoral, també es poden prendre les dades amb un dispositiu molt més còmode col-locat al dit de la mà.

\section{- BIOFORCE HRV}

http://www.bioforcehrv.com/

Aquesta aplicació incorpora les mateixes funcions que la resta: controla el sobreentrenament, optimitza els entrenaments, i monitora els canvis setmanals i mensuals amb una plataforma gràfica de molta qualitat.

\section{- FIRSTBEAT}

https://www.firstbeat.com/

És un software comercial d'un perfil diferent dels anteriors; no està adreçat directament a aplicacions de dispositius mòbils, sinó que és compatible amb la majoria de pulsímetres comercials (Suunto ${ }^{\mathrm{TM}}$, Garmin ${ }^{\mathrm{TM}}$, Samsung ${ }^{\mathrm{TM}}$, Sony ${ }^{\mathrm{TM}}$, etc.) i utilitza les seves dades. A part de les funcions típiques d'anàlisi de la VFC com són l'equilibri estrès/recuperació, també incorpora algoritmes que estimen la despesa metabòlica i el consum màxim d'oxigen $\left(\mathrm{VO}_{2 \max }\right)$.

\section{- ELITEhrv}

https://team.elitehrv.com

És una aplicació centrada en el rendiment esportiu basat en l'equilibri simpàtic/parasimpàtic, que incorpora aspectes com l'estat d'ànim i la qualitat de la son per completar el diagnòstic.

\section{- HRV4Training}

http://www.hrv4training.com/

És una aplicació de gestió de l'entrenament molt similar a les anteriors però amb una sèrie de particularitats. En primer lloc, actualment només està disponible per a dispositius iPhone ${ }^{\mathrm{TM}} \mathrm{i} / \mathrm{o} \mathrm{Pad}^{\mathrm{TM}}$. Utilitza la càmera del telèfon per a extreure les dades de temps de l'interval RR; la tècnica es diu fotopletismografia i consisteix en la detecció de canvis en el volum sanguini durant un cicle cardíac, mitjançant la illuminació de la pell i en la mesura dels canvis amb l'absorció de la llum.

\section{- CARDIOMOOD}

http://www.cardiomood.com/en/

Segurament és l'aplicació menys especialitzada en l'àmbit de l'esport. Fa una anàlisi de la VFC, però els resultats que mostra els relaciona amb l'energia vital, ja siguin producte de l'estrès laboral o o de l'estrès esportiu, donant unes recomanacions molt generals sobre l'activitat a desenvolupar.

\section{Conclusions}

El seguiment de la VFC s'ha generalitzat cada cop més en l'àmbit de l'entrenament, tant a nivell individual com en equips. Els instruments per a l'anàlisi de la VFC són cada cop més nombrosos i sofisticats.

El problema més gros amb el qual ens podem trobar és caure en una interpretació massa simplista de les dades. En primer lloc no podem extreure conclusions d'un sol registre de VFC. És a dir, si analitzem les dades d'un sol registre d'un individu, no podrem comparar-les amb res; per tant, necessitem una sèrie de registres per a observar l'evolució i, d'aquesta manera, detectar alguna mena de tendència i poder treure conclusions.

Per altra banda, un cop detectada aquesta tendència, caldria veure si durant el període observat hi hagut molta variació entre els registres, és a dir, si han fluctuat molt entre mesura i mesura, ja que aquest extrem ens indica les adaptacions del nostre cos que s'han anat produint durant el període observat. Per exemple, una tendència que indiqui la disminució de la VFC, que és una resposta típica a un factor d'estrès, no ha de ser necessàriament un element d'alarma; tampoc no ha de ser un símptoma de sobreentrenament; simplement pot ser fruit d'una sobrecàrrega típica del procés d'entrenament i que tindrà la seva sobrecompensació en el moment en què el cos recuperi la seva homeòstasi.

I, per últim, cal tenir en compte altres factors de la vida quotidiana que poden influir en aquest fet, com, per exemple, la qualitat del son, l'estrès generat per exàmens o feina, que poden influir en els resultats obtinguts en els registres de VFC. 


\section{Referències}

Aubert, A. E., Seps, B., \& Beckers, F. (2003). Heart rate variability in athletes. Sports Medicine, 33(12), 889919.

Cottin, F., Medigue, C., Lepretre, P.-M., Papelier, Y., Koralsztein, J.-P., \& Billat, V. (2004). Heart rate variability during exercise performed below and above ventilatory threshold. Medicine and Science in Sports and Exercise, 36(4), 594-600.

D’Ascenzi, F., Alvino, F., Natali, B. M., Cameli, M., Palmitesta, P., Boschetti, G., ... Mondillo, S. (2014). Precompetitive assessment of heart rate variability in elite female athletes during play offs. Clinical Physiology and Functional Imaging, 34(3), 230-236.

Dupuy, O., Bherer, L., Audiffren, M., \& Bosquet, L. (2013). Night and postexercise cardiac autonomic control in functional overreaching. Appl Physiol Nutr Metab., 38(2), 200-208. http://doi.org/10.1139/ apnm-2012-0203

Flatt, A. A., \& Esco, M. R. (2013). Validity of the ithleteTM smart phone application for determining ultra-short-term heart rate variability. Journal of $\mathrm{Hu}$ man Kinetics, 39(1), 85-92.

Franchini, E., de Moraes Bertuzzi, R. C., Takito, M. Y., $\&$ Kiss, M. a P. D. M. (2009). Effects of recovery type after a judo match on blood lactate and performance in specific and non-specific judo tasks. European Journal of Applied Physiology, 107(4), 377-383. http:// doi.org/10.1007/s00421-009-1134-2

Gamelin, F. X., Berthoin, S., \& Bosquet, L. (2006). Validity of the polar S810 heart rate monitor to measure RR intervals at rest. Medicine and Science in Sports and Exercise, 38(5), 887.

Hynynen, E., Konttinen, N., Kinnunen, U., Kyröläinen, H., \& Rusko, H. (2011). The incidence of stress symptoms and heart rate variability during sleep and orthostatic test. European Journal of Applied Physiology, 111(5), 733-741.

Kaikkonen, P., Hynynen, E., Mann, T., Rusko, H., \& Nummela, A. (2010). Can HRV be used to evaluate training load in constant load exercises? European Journal of Applied Physiology, 108(3), 435-442.

Kaikkonen, P., Hynynen, E., Mann, T., Rusko, H., \& Nummela, A. (2012). Heart rate variability is related to training load variables in interval running exercises. European Journal of Applied Physiology, 112(3), 829-838.

Kaikkonen, P., Nummela, A., \& Rusko, H. (2007a). Heart rate variability dynamics during early recovery after different endurance exercises. European Journal of Applied Physiology, 102(1), 79-86. http://doi. org/10.1007/s00421-007-0559-8

Kaikkonen, P., Nummela, A., \& Rusko, H. (2007b). Heart rate variability dynamics during early recovery after different endurance exercises. European Journal of Applied Physiology, 102(1), 79-86.

Kotov, A. V, \& Revina, N. E. (2012). Heart Rate Variability During «Alarm Stage» of Burnout Syndrome in Emergency Doctors. Bulletin of Experimental Biology and Medicine, 1-3.
Morales, J., Álamo, J. M., García-Massó, X., Buscà, B., López, J. L., Serra-Añó, P., \& González, L.-M. (2014). Use of heart rate variability in monitoring stress and recovery in judo athletes. Journal of Strength and Conditioning Research, 28(7), 1896-1905.

Morales, J., Garcia, V., García-Massó, X., Salvá, P., Escobar, R., \& Busca, B. (2013). The use of heart rate variability in assessing precompetitive stress in highstandard judo athletes. Int J Sports Med, 34(2), 144151.

Nunan, D., Donovan, G. A. Y., Jakovljevic, D., Hodges, L., Sandercock, G., \& Brodie, D. (2009). Validity and reliability of short-term heart-rate variability from the Polar S810. Medicine+ Science in Sports+ Exercise, 41(1), 243.

Pichon, A. P., de Bisschop, C., Roulaud, M., Denjean, A., \& Papelier, Y. (2004). Spectral analysis of heart rate variability during exercise in trained subjects. Medicine and Science in Sports and Exercise, 36, 17021708.

Plews, D. J., Laursen, P. B., Stanley, J., Kilding, A. E., \& Buchheit, M. (2013). Training adaptation and heart rate variability in elite endurance athletes: opening the door to effective monitoring. Sports Medicine, 43(9), 773-781.

Rodas, G., Pedret, C., Ramos, J., \& Capdevila, L. (2008a). Variabilidad de la frecuencia cardíaca: concepto, medidas y relación con aspectos clínicos (I). Archivos de Medicina Del Deporte: Revista de La Federación Española de Medicina Del Deporte Y de La Confederación Iberoamericana de Medicina Del Deporte, (123), 41-48.

Rodas, G., Pedret, C., Ramos, J., \& Capdevila, L. (2008b). Variabilidad de la frecuencia cardiaca: conceptos, medidas y relación con aspectos clínicos (parte II). Archivos de Medicina Del Deporte: Revista de La Federación Española de Medicina Del Deporte Y de La Confederación Iberoamericana de Medicina Del Deporte, (124), 119-127.

Sartor, F., Vailati, E., Valsecchi, V., Vailati, F., \& La Torre, A. (2013). Heart rate variability reflects training load and psychophysiological status in young elite gymnasts. Journal of Strength and Conditioning Research / National Strength \& Conditioning Association, 27(10), 2782-90. http://doi.org/10.1519/JSC. Ob013e31828783cc

Seiler, S., Haugen, O., \& Kuffel, E. (2007). Autonomic recovery after exercise in trained athletes: intensity and duration effects. Medicine and Science in Sports and Exercise, 39(8), 1366.

Stein, P. K., Barzilay, J. I., Chaves, P. H. M., Mistretta, S. Q., Domitrovich, P. P., Gottdiener, J. S., ... Kleiger, R. E. (2008). Novel measures of heart rate variability predict cardiovascular mortality in older adults independent of traditional cardiovascular risk factors: the Cardiovascular Health Study (CHS). Journal of Cardiovascular Electrophysiology, 19(11), 1169-1174.

Task Force of the European Society of Cardiology and the North American Society of Pacing and Electrophysiology. (1996). Standards of measurement, physiological interpretation, and clinical use. Task 
Force of the European Society of Cardiology and the North American Society of Pacing and Electrophysiology. Circulation, 93, 1043-1065.

Thiel, C., Vogt, L., Bürklein, M., Rosenhagen, A., Hübscher, M., \& Banzer, W. (2011). Functional Overreaching During Preparation Training of Elite Tennis Professionals. Journal of Human Kinetics, 28(-1), 79-89. http://doi.org/10.2478/v10078-011-0025-X

Weippert, M., Kumar, M., Kreuzfeld, S., Arndt, D., Rieger, A., \& Stoll, R. (2010). Comparison of three mobile devices for measuring R-R intervals and heart rate variability: Polar S810i, Suunto t6 and an ambulatory ECG system. European Journal of Applied Physiology, 109(4), 779-786.

Yamamoto, Y., Hughson, R. L., \& Peterson, J. C. (1991). Autonomic control of heart rate during exercise studied by heart rate variability spectral analysis. Journal of Applied Physiology, 71(3), 1136-1142.

\section{Soluciones tecnológicas para el estudio de la variabilidad de la frecuencia cardíaca, el estrés i la recuperación}

Resumen. La variabilidad de la frecuencia cardíaca (VFC) hace referencia al tiempo del intervalo entre latido y latido del corazón. Debido a que este intervalo no es fijo, sino que oscila constantemente, la dinámica de estas oscilaciones junto con un análisis matemático adecuado puede aportar una información muy valiosa sobre el equilibrio del Sistema Nervioso Autónomo (SNA) como responsable del funcionamiento del corazón. El análisis de la VFC aporta información sobre cualquier situación que interfiera el equilibrio homeostático en nuestro organismo; es por ello que su variación va asociada con la presencia de ciertas patologías. En el ámbito del entrenamiento de los deportes, dado que el ejercicio físico es un agente promotor del estrés fisiológico, los cambios de la VFC se han asociado a la asimilación de las cargas de entrenamiento o a ciertas condiciones vinculadas al síndrome de sobreentrenamiento. Los avances tecnológicos han aportado soluciones para analizar la VFC; en este sentido se presentan diferentes opciones de software científico para analizar en profundidad la dinámica de la VFC, así como diversas opciones de aplicaciones para dispositivos móviles que cada vez más ocupan este espacio en el mundo del entrenamiento deportivo.

Palabras clave: Sistema nervioso autónomo; sobreentrenamiento; Intervalo inter-pulsación; equilibrio simpático-parasimpático 